\title{
Rethinking Screen Time during COVID-19: Impact on Psychological Well-Being in Physiotherapy Students
}

\author{
Isha Akulwar-Tajane, ${ }^{1, *}$, Kashish K. Parmar², Palak H. Naik², Ayushi V. Shah ${ }^{2}$ \\ ${ }^{1}$ Neuro Physiotherapy, K J Somaiya college of Physiotherapy, Mumbai, India. \\ ${ }^{2} \mathrm{~K}$ J Somaiya College of Physiotherapy, Mumbai, India.
}

How to cite this paper: Isha Akulwar-Tajane, Kashish K. Parmar, Palak H. Naik, Ayushi V. Shah. (2020) Rethinking Screen Time during COVID-19: Impact on Psychological Well-Being in Physiotherapy Students. International Journal of Clinical and Experimental Medicine Research, 4(4), 201-216.

DOI: $10.26855 /$ ijcemr.2020.10.014

Received: August 17, 2020

Accepted: September 10, 2020

Published: September 24, 2020

*Corresponding author: Isha Akulwar-Tajane, Neuro Physiotherapy, K J Somaiya college of Physiotherapy, Mumbai, India.

Email: drishasa@yahoo.co.in

\begin{abstract}
We do not just use technology; we live with it. Much more deeply than ever before, we are aware that interacting with technology involves us emotionally, intellectually, and sensually. As the popularity of the internet and digital devices grows so do concerns over their excessive use. This is worth drawing attention to, though, considering the widely established consequences excessive screen time can have on someone's sleep, mental and physical health. This paper describes the pattern of screen use, sleep and psychological well being in physiotherapy students during the COVID-19 pandemic lockdown. The study also throws light on the fact that physical exercises could be a mediating factor for effect on sleep pattern and overall perception of well-being. A representative sample of 150 Physiotherapy students was surveyed to assess technology use, sleep pattern, mental and physical health through a self-reported questionnaire. Around 52\% students admitted that excessive screen use has affected their sleep quality and linked it to sleeping problems such as reduced sleep durations with mobile phone use-awakenings. $48 \%$ of the students believe that excessive hours spent in front of the screen has affected their physical well being and $28.7 \%$ of students reported negative effects on their mental health. $65.27 \%$ of the exercising participants experienced beneficial effects due to performance of physical exercises and reported decreased sleeping complaints or mental health issues. The present study strengthens the existing scientific evidence for the negative impact of excessive screen time on mental and physical health while providing new insights on the digital media use during pandemic situation. In addition, this study also attempts to determine the mediating effect of physical exercise on ill effects of screen use.
\end{abstract}

\section{Keywords}

COVID-19 pandemic, lockdown, screen use, sleep, physical health, mental health, psychological well being, physiotherapy students, physical exercise

\section{Introduction}

Within the constructs of civilized society, the vast rewards of technological innovations have led to the widespread use of portable electronic devices and the normalization of screen media devices. Smartphones have become 
an indispensable part of modern life since they are used for various purposes including communication, leisure time entertainment, social networking, information gathering, navigation, workplace applications, and so forth. There are $>950$ million smartphone users in India alone [1]. The Technology Acceptance Model (TAM) is the theoretical construct which explains the reasons behind adoption of smartphones, as being lightweight, trendy, multi-functional, portable, customizable, and user-friendly, are obvious contenders for a higher perceived usefulness and perceived ease of use compared to other gadgets [2] [3]. Technological advancements have impacted every one of us and can give us an edge in our personal and professional lives. Numerous studies in educational research have shown that the use of mobile devices is widely adopted by the medical professionals, especially the young trainees with perceived benefits in education, patient care, communication, and research [4] [5] [6] [7].

However, given the benefits of technology, there exist a number of negative effects too. This leads us to ask the question, "What are some negative effects of technology"? The concept is under significant research with related concepts in digital media use and mental health.

Debate about the psychological impacts of screen time on young people remains a regular feature of societal conversation. Specifically, concerns that screen time negatively impacts psychosocial functioning, health and behaviours remain widespread, and are routinely voiced in academic, policy and media circles. Whilst some evidence routinely shows negative correlations between screen time and well-being [8] [9], recent reviews and meta-analyses repeatedly conclude that the evidence base is difficult to interpret and presents mixed findings [10] [11]. In recent years, a growing consensus has emerged suggesting that the associations observed between screen time and psychological or health outcomes are very small in size and that there lacks clear longitudinal evidence that they represent a pure causal effect driven by screen media [12] [13] [14]. Strong evidence concerning the positive or negative impacts of screen time is, therefore, surprisingly sparse.

Smartphones, laptops, and all kinds of digital devices incorporate the use of the internet. The increasing use of and dependence on the internet brought to the surface a new public health concern near the start of the century "Internet Addiction Disorder", which was eventually included in the Diagnostic and Statistical Manual of diseases (DSM-5) under conditions for further study [15] [16]. On the heels of this, the close association between the internet and the smartphone led to the introduction of a new condition called "Smartphone Addiction" [17], and has become a more serious problem. Reported in other terms like "Problematic Smartphone Use" and "Smartphone Use Disorder", this is a behavioral addiction characterized by the presence of symptoms of tolerance, salience, mood changes, and dependence on smartphones.

Smartphone abuse is increasing in the 21st century as more and more people enjoy exploring smartphones in their free hours. Researchers have evaluated individuals' subjective smartphone usage and reported prevalence of smartphone addiction ranging from $8.7 \%$ in Korea to 32\% in India [18] [19]. A recent Deloitte study found that $60 \%$ of U.S. adults ages 18-34 admitted to smartphone overuse [20]. There is no denying that an individual's screen time has increased exponentially in the past decade. In fact, a 2018 Nielsen report [21] found that American adults $\log$ a total of about 11 hours of screen time a day. Young adults $18-34$ spend $43 \%$ of their time consuming media on digital devices. Behind this surge are the growing use of new platforms, as well as the younger, multicultural generations who leverage them. Almost a third of their time spent with media (29\%) comes from apps/web on a smartphone - the most of any measured generation. As per a recent survey by smartphone brand Vivo, in association with Cybermedia Research (CMR), an average Indian is spending one-third of his or her waking hours on the phone and the dependency over smartphones has increased. All forms of screens are most frequently used by younger age brackets, particularly college students. In the academic realm, smartphone abuse to addiction is becoming a rapidly emerging issue because most students whether higher learning students or low level students are addicted to applications found on smartphones. 2019 International Journal of Environmental Research and Public Health study found college-age individuals already spend an average of between eight and 10 hours a day on smartphones alone. Recent studies have revealed a high prevalence of smartphone addiction in university students, ranging from 34\% to $40 \%$ in medical students across different states of India [19] [22] [23] [24]. Yet another Indian study showed that $40 \%$ of postgraduate residents using a smartphone fulfilled the criteria for smartphone dependence [25].

Smartphone addiction is a global public health concern and has been extensively researched in the adolescents population. Similar to other addictions (substance and behavioral), excessive and addictive smartphone use has been linked to life stress, lower self-efficacy, higher perceived stress, high internal locus of control, materialism, and internet addiction [26]. It has been shown that young people who use cell phones excessively (for both speaking and text messaging) have increased restlessness, more careless lifestyles, greater consumption of stimulating beverages and greater susceptibility to stress [26]. The study of 200 adolescents in Korea also showed that abnor- 
mal users of smartphones had significantly more problematic behaviors, somatic symptoms, attention deficits, and aggression [27]. The pattern of use with a cellular phone includes risky behavior such as its use while driving, on silent mode, in closed spaces and while charging.

New research suggests that excessive use of smartphones, increases the risk for severe psychopathologies and there is growing evidence of problematic use of smartphones that impacts both social and health aspects of users' lives [27] [28]. The time spent sitting and viewing a screen has been linked to mental health effects such as anxiety and depression [29] [30]. With this added risk, lack of sleep plays a major role in a healthy mindset, but without proper rest, mental health can degrade at a higher risk. Sleeping helps us to recover from mental as well as physical exertion and is vital for maintaining good mental and physical health. Overall, sleep helps humans maintain optimal emotional and social functioning while we are awake by giving rest during sleep to the parts of the brain that control emotions and social interactions [31]. Inadequate rest impairs the ability to think, to handle stress, and to moderate emotions. Sleep disturbance is considered as the second most common symptom of mental distress [32]. More recent views suggest that sleep can play a causal role in both the development and maintenance of different mental health problems. Medium and long term effects of insomnia have been linked to the psychiatric problems - depression, anxiety conditions, alcohol and other substance abuse [33]. Whereas the medical consequences of chronic sleep deprivation are — cardiovascular, respiratory, renal, gastrointestinal, musculoskeletal disorders, impaired immune system function; and an increased risk of mortality.

Epidemiological studies have already demonstrated high prevalence of sleep complaints with as much as one third of the adult population reporting difficulty sleeping [34] [35] [36]. In medical students, poor sleep quality, sleep related problems and abnormal sleep habits have been reported with a prevalence ranging from $37 \%$ to $59 \%$. [37] [38] [39] [40].

Excessive mobile phone use has been linked to sleeping problems in cross-sectional studies in adults. Generally a linear relationship with detrimental effects on sleep pattern and general health with increasing media use has been reported [41]. Moreover, screen time at night keeps adults from falling asleep and sleeping well due to cognitive stimulation and sleep deprivation. Much of the effect of screen time comes from the fact that people don't do more healthful activities as a result - they sleep and exercise less, and they eat more leading to obesity and associated risk of other comorbidities. Lifestyle influences from increasing access and use of screen-based media devices have been shown to contribute heavily to the detrimental sleep hygiene of individuals of any age group and also sedentary behavior. Young adults make extensive use of social media devices that interact with daily activities, including sleep [42].

When it comes to impact on health, physicians are increasingly seeing patients who exhibit signs of technology overuse, particularly with the current reliance on smartphones in our daily lives.

A substantial number of studies in medical students who used a smartphone reported possible health hazards. These include headache, sleep disturbance, lack of concentration, impairment of short-term memory, dizziness, heating of the ear, burning skin, brain tumors and hypertension [43]. Moreover, mobile phones have been reported to cause discomfort, general ill-being, nausea, muscular pain, forgetfulness and fatigue [44]. In yet another study on medical female students, the main effects of smartphone use were reported to be on memory, sleep and concentration, which might affect academic performance [45]. No reports on health issues related to the use of the smartphones in the population of physiotherapy students have been published yet, although they are used excessively by them. Our goal was therefore to study the pattern of use of smartphones and related health hazards among physiotherapy students.

\section{Context and Relevance of the Study}

Confined in homes during COVID-19 lock down, we are living through a period where technology is in many regards the only connection to normalcy. It means using technology to move forward when life seems to be on pause. After all, screens are the reason why students can still pursue their education, continue study work, and keep in touch with friends and family when they can't leave their homes. While some forms of technology may have made positive changes in the world; sociologists, media theorists and technology experts have argued that digital technology is distracting us, resulting in negative social and emotional consequences. The explosion of technology and excessive screenification in this unprecedented situation has many apprehensions about the pandemic's negative impact on the vulnerable population of young adults. Some preliminary reports indicate that there has been a drastic increase in the screen time usage during the lockdown which can be predicted to be over and above the impact of college day social media use as focused in the previous studies. Also due to significant disruption in routine, nocturnal media use has been increased. As more students enjoy exploring their smartphones in their free hours, an 
increasing reliance on cell-phones among young adults and college students may signal the evolution of cell-phone use from a habit to an addiction in this lock down situation. This is worth drawing attention to, though, considering the widely established consequences excessive screen time can have on someone's sleep, mental and physical health.

Over the last 3 months of the COVID-19 pandemia sleep changes are just one of the innumerable daily activities that have been impacted by the virus. Anxiety spurred on by the pandemic, seeping into dreams, and messing up sleep patterns is a common occurrence for millions of adults globally, according to the preliminary surveillance reports. This is of concern, since sufficient sleep is crucial for healthy somatic, cognitive and psychological well-being.

Psychological stress is a normal reaction to a threat or disturbing change in the environment. According to some experts in psychiatry, mental health issues like anxiety, depression have been on a historic rise in the major pandemic which the world is facing. After the Covid-19 outbreak, during lockdown 1.0 itself, the Indian Psychiatric Society [46] reported that mental health concerns have escalated by an alarming $20 \%$, with an unprecedented rise in psycho-social concerns like: family issues, depression, anxiety disorders, feelings of suicide, substance abuse, sexual abuse and domestic violence. While reporting similar issues South African Depression and Anxiety Group (SADAG) study found that many people are dealing with a combination of issues, including the spread of the virus, finances, relationship problems, job security, grief, gender-based violence and trauma [47].

Given the recency of the pandemic, studies related to this technological infiltration in the student population do not yet exist. Additional information in this area may be helpful for educators who are looking to determine the appropriate course of action for students. Thus the present study aims to unravel the effects of excessive mobile-phone use and screen time on new occurrence of sleep problems and general health symptoms in students during COVID-19 pandemic lock down. Although the associations between electronic media use and sleeping problems seem quite consistent in the literature, the mediating effect of physical activity has not been well studied in this context. Thus, another purpose of this study is to determine if physical exercises have a positive effect on sleep and mental health disorders which have occurred during COVID-19 lockdown. From the educator's perspective, this study will guide us to offer solutions to mitigate the impact of these problems on health in general which might have repercussions on the academic performance of students as well.

The specific objectives outlined in the study are: 1 . to analyze the pattern of digital device use during lockdown; 2. to analyze the effects of screen time on physical health and mental well-being; 3 . to determine if exercises have a positive effect on sleep disorders and mental and physical health problems.

\section{Methodology}

Participants: The current study was conducted among university students attending K.J. Somaiya College of Physiotherapy, Mumbai, India. Undergraduate (I-IV year) Physiotherapy students, interns, postgraduate students enrolled in the institute at the time of study were invited to participate in an online survey. Students were excluded if: (a) they refused to participate in the study; (b) had any health problem that temporarily or permanently prevented participation in physical activity; (c) used any type of medication that could induce changes in the study variables (e.g., opioids or antidepressants).

Procedure: It was a cross-sectional qualitative survey conducted during COVID-19 lock down period (April to June 2020). Ethical approval was obtained from the institutional review board of K.J. Somaiya college of Physiotherapy, India. Participation in the study was voluntary and electronic consent was obtained from the participant. The target population was recruited by a non-randomized convenient sampling method and is representative of students available on social media platforms. Sample size was not estimated prior to the study. However, a maximum number of participants were desirable as well as anticipated in view of relevance of this topic to students in the current situation; and the beneficial use of social media as a method of data collection.

Measures: A questionnaire was developed de-novo as a part of this study. The questionnaire includes student's academic profile details and other sociodemographic and behavioural characteristics of including age, sex, exposure to confounding factors such as caffeine consumption, alcohol consumption, smoking and exposure to second-hand smoking, etc. Students self-reported their usage pattern of any screen-based mobile device (mobile phone, tablet, laptop, television etc.). Sleep variables were derived from self-reported sleep patterns pertaining to sleep latency, sleep duration, use of sleeping medication, daytime dysfunction, and quality of sleep. Sleep quality was assessed using four standardized dimensions from the Swiss Health Survey [48]: difficulty falling asleep, sleeping restlessly, waking up several times during the night and waking up too early in the morning [48]. Students were asked how often they have encountered these sleep quality problems during the last 2 months using a 
four-point Likert scale (Never, Rarely, Sometimes, and Often). The questionnaire also sought to obtain perspectives of students on the impact of screen use on their physical health, mental health. To design the questionnaire, review of information was done from relevant preliminary reports, blogs, etc. to construct the overall picture of social and psychological experience during the lockdown period.

The questionnaire is self-administered and in English language. It is semi-structured with a combination of open and close ended-questions (includes multiple choice and ranking Likert-scale style questions). Content validity of the questionnaire was established from two experienced Physiotherapy teachers. The questionnaire was distributed to the participants as Google forms via social media on Whatsapp; and was emailed, if requested by them. Link to the forms was available to them for a period of one week. Reminders were sent to ensure maximum participation.

Data thus collected was subjected to analysis.

\section{Data Analysis}

All the responses obtained via Google-forms were screened. Inaccurate or incomplete responses were discarded from the analysis. The close-ended data was analyzed automatically using Google spread-sheet and descriptive statistics using percentage and frequency distribution was performed. The open-ended questions were analyzed using categorization and thematic analysis.

\section{Results}

Approximately one third of the invited participants completed the survey. A total number of 150 completed forms were analyzed. This study sample is representative of students from all academic years from I B.P.Th. to IV B. P. Th. and interns and postgraduate students. The age of the participants was $20.15 \pm 1.46$ years (mean \pm SD) and $92 \%$ were females.

\subsection{Screen Time Use in COVID-19 Lockdown}

Screen time is the amount of time that is spent using a device such as a smartphone, computer, television, tablet, iPhone, laptop, or video game console [49]. Table 1 and accompanying pie charts (Figure 1 and Figure 2) show the usage pattern of digital devices during the lockdown by study participants.

All the participants reported using at least one screen based mobile device, smartphone being the commonest device followed by television and other portable digital devices. It can be seen that with the evolution of mobile phones to smartphones and by encompassing all of the internet features and mobile applications, the technological usage discussed above have been shifting gradually to smartphones. Multiple device use in varied combinations is also reported by the participants.

Table 1. Usage pattern of digital devices in the lockdown by the participants

\begin{tabular}{cccccc}
\hline Mobile phone & Tablet & Laptop & Computer & Video game console & Television \\
\hline $98 \%$ & $7.3 \%$ & $28 \%$ & $3.3 \%$ & $1.3 \%$ & $48.7 \%$ \\
\hline
\end{tabular}
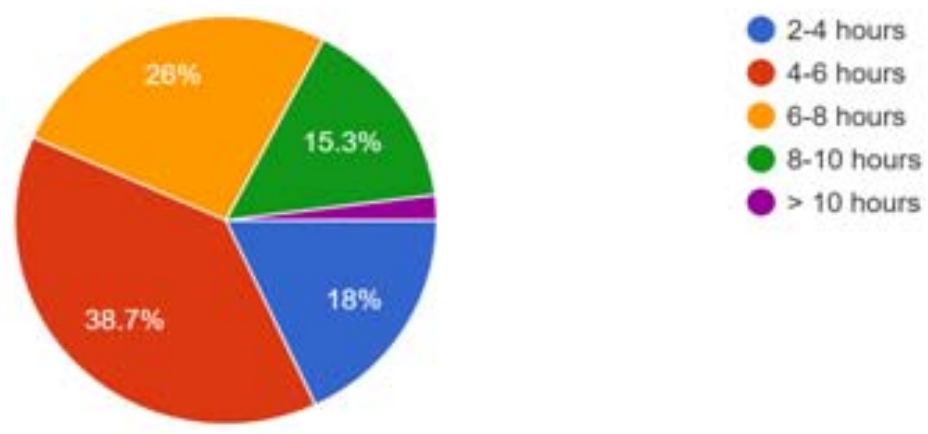

Figure 1. The above pie chart depicts the amount of hours spent on the digital devices by the participants. 


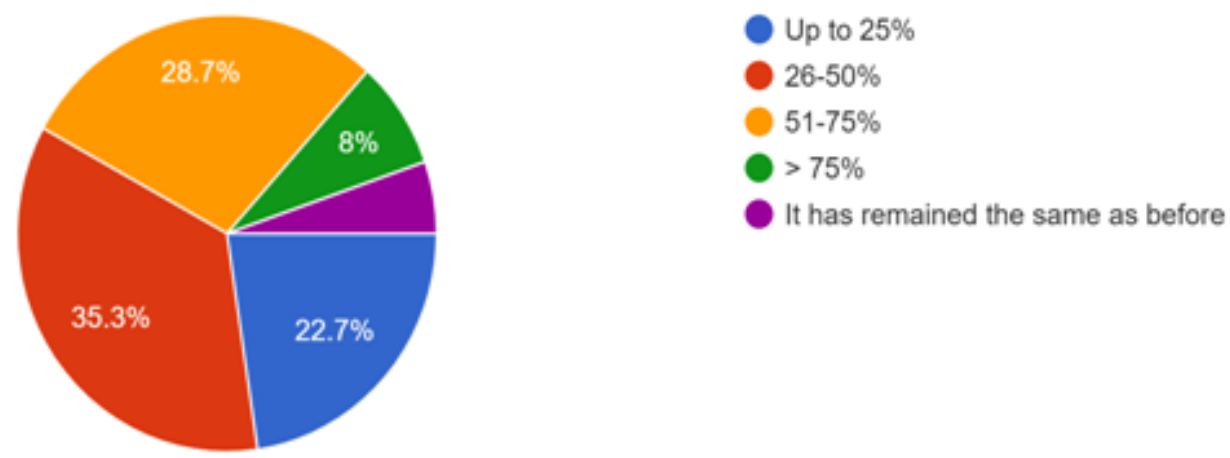

Figure 2. The above pie chart shows by how much the screen time has increased during the lockdown.

For $94.7 \%$ students screen time use had increased during the lockdown, the extent of increase being moderate (51\%-75\%) or high (> 75\%) for nearly two thirds of the population. $43.3 \%$ of student participants spent more than 6 hours on digital devices on a daily basis. Due to significant disruption in routine during lock down, nocturnal media use has also been increased. The vast majority of students have access to the internet, and many have social media profiles. The digital media activities involved computer work, Email/instant messaging, social networking sites, video games, surfing the internet, video calls/calls on phones, watching online videos, reading e-books, watching TV/DVDs, and watching movies, TV series, etc. Predominant means of use was entertainment for $40 \%$ students, educational purposes for $34 \%$ of students and $30.6 \%$ for social networking and communication (30.6\%).

These findings are in accordance with some preliminary reports suggesting that during the pandemic Tech use has increased tremendously among the younger generation and students. With more people having to stay at home, people's use of the internet for entertainment and social networking accelerate globally as evident from the statistical metrics published by various commercial media agencies such as YouTube, Zoom, Amazon, Netflix, Facebook, digital game distributors, etc., and other national broadband networks and mobile applications [50] [51] [52]. In the current situation of social distancing, we can harness our capacity to interact socially online to enable social support and social connectedness, and tackle loneliness, whilst physically distancing ourselves from others. Research shows that social media and video games provide temporary escape from real life and offer valuable social engagement [50]. On the other hand, sociologists, media theorists and technology experts have argued that digital technology is distracting us, resulting in negative social and emotional consequences.

It is an unusual situation for university students to have some free time in hand. Under the extended COVID-19 lockdown, while there is anxiety in the atmosphere, there is also potentially a quiet time for contemplation and for a focus on personal growth. Students can find themselves staring at an opportunity to work on themselves, to work on a self-development plan. Along with other purposes, use of screen time for various personal and professional skill development activities reported by $34 \%$ of the students is a promising behavior towards their education.

\subsection{Mental Health and Well Being}

Majority of the participants admitted to have developed media addiction during this lock down phase. Addiction is a process whereby a behavior that can function both to produce pleasure and to provide an escape from internal discomfort is employed in a pattern characterized by: (1) recurrent failure to control the behavior (powerlessness); and (2) continuation of the behavior despite significant negative consequences (unmanageability) [53].

Prior research on excessive screen media or smartphone use typically employs the amount of time spent on smartphones or apps as the sole indicator of excessive use [54]. However, recent conceptualizations of excessive use are based on symptoms of addiction to or dependence on screen media and problems in functioning; and offer an alternative way to quantify problematic phone use. Specifically, the inclusion of Internet Gaming Disorder (IGD) in the Diagnostic and Statistical Manual of Mental Disorders 5th Edition (DSM-5; American Psychiatric Association [APA], 2013) provides a set of nine symptoms that captures disordered gaming (video game addiction) and other screen-media addictions such as Social Media Dependence [55] [56]. Smartphone addiction is measured with these nine symptoms: tolerance (needing to use Smartphone for longer amounts of time to get same effect), withdrawal, preoccupation, loss of interest in other activities, difficulty controlling use, conflict over use, using smartphone to escape problems or negative effect, deception, psychosocial problems due to use, and substantial conflicts with others (impacting one's relationships) due to use. 
Stated below are some of the quotes from the participants that indicate a trend towards media addiction and lack of self-control:

P16: "I feel the urge to constantly check my social networking apps". P71: "I have become addicted to my Phone. I feel the need to check it in a while". P98: "I reflect on what I have seen continuously". P103: "Due to stress of studies I tend to check my phone often as an escape".

As more students enjoy exploring their smartphones in their free hour, an increasing reliance on cell-phones among young adults and college students may signal the evolution of cell-phone use from a habit to an addiction in this lock down situation. Though we did not employ any smartphone addiction scale, this compulsive nature of checking smartphones frequently indicates a trend towards smartphone addiction. Smartphone users also reported feelings of extreme anxiety and cognitive delays on separation from their smartphones. Some work has measured the effects of smartphone abstinence (for up to 24 hours) on short-term outcomes such as mood, anxiety and craving and reported similar findings [57] [58]. These findings highlight the effects of smartphone overuse on cognitive control and emotion-related dysfunctional coping processes related to screen media use.

When asked about if and how they regulate screen time use, only 18\% reported that they keep a track of screen use every day, some of them use mobile based applications such as digital well-being for the same. Also, good technology behavior was practiced by some students as they replace some of the unnecessary usage with a physical activity (35.3\%), or with family get-together (52.7\%) and do not use it while studying $(0.7 \%)$.

It's alarming to see the various mental health issues experienced by the study participants during lock down (Table 2). $91.3 \%$ of the participants reported emotional issues such as low mood, anxiety, irritability, erratic behavior, along with poor cognitive functioning and performance (e.g., forgetfulness, making mistakes and slower thinking than normal). Restlessness, uncertainty of the whole situation, COVID-19 crisis, uncertainty of exams, emotional imbalance, overthinking, etc., were their subjective feelings. $28.7 \%$ of the study participants reported that screen time had negatively affected their mental health. The positive or negative health effects or screen time are influenced by levels and content of exposure. In the last decade, increasing mental distress and treatment for mental health conditions among young adults has paralleled a steep rise in the use of smartphones and social media. A temporal trend with increased use in screen time has been shown to be directly correlated with an increased chance of depression in adults. Adults who spend six hours or greater using screen time are more likely to suffer from moderate to severe depression [29]. Whereas in a study by NCBI [59], an univariate analysis between screen time and depression indicated that adults who spent $>4 \mathrm{~h}$ per day in watching TV and using the computer, are more likely to be diagnosed with moderate or severe depression than those who spent $<4 \mathrm{~h}$ per day in watching TV. In a study in Chinese college freshmen when screen time $>2 \mathrm{~h}$ /day was compared with screen time $\leq 2 \mathrm{~h} /$ day, the OR was 1.38 for anxiety, 1.55 for depression and 1.49 for psychopathological symptoms [60]. In the current study, depression was reported by $15.3 \%$ of students while $43.3 \%$ were spending more than 6 hours actively on digital devices.

Table 2. Mental health issues reported by the participants

\begin{tabular}{cc}
\hline Symptom & Percentage of participants \\
\hline Mood swings & $67.3 \%$ \\
Less motivation & $65.3 \%$ \\
Frustration & $53.3 \%$ \\
Mental breakdown due to no specific reason & $38.7 \%$ \\
Anxiety & $38.7 \%$ \\
Social isolation & $22 \%$ \\
Depression & $15.3 \%$ \\
Scared/panic & $0.7 \%$ \\
None & $8.7 \%$ \\
\hline
\end{tabular}

Screen use releases dopamine in the brain, which can negatively affect impulse control [61]. Studies have shown screen time affects the frontal cortex of the brain, similar to the effect of cocaine. Similar to drugs, screen time sets off a pleasure/reward cycle that can have a negative impact on your life. Cognitive neuroscientists have shown that rewarding social stimuli-aughing faces, positive recognition by our peers, messages from loved ones-activate the same dopaminergic reward pathways. Smartphones have provided us with a virtually unlimited supply of social 
stimuli, both positive and negative. Every notification, whether it's a text message, a "like" on Instagram, or a Facebook notification, has the potential to be a positive social stimulus and dopamine influx. Screen time thus, acts like a digital drug for the brain.

The screen content of social media is known to stir emotions and has been linked with psychological and somatic arousal; and cognitive over-activation through the media content [62].

In the current unprecedented situation, it is likely that similar to the general population, students were using the internet to obtain health information related to COVID-19 and its associated consequences. These healthy individuals may be seeking information about this novel disease, educating themselves about diseases in the news or looking into other health-relevant concerns. Additionally, those with unmeasured psychosomatic illnesses or those more prone to health anxiety may have self-selected online health resources. Reading about disease might increase people's health anxiety, reinforce hypochondriasis, or cause them unnecessary concern about the health status of themselves or loved ones. Given this possibility of inducing unnecessary health concerns and that some poor quality health information is available online; the use of online health information by healthy people may harm their psychological well-being. In a longitudinal study by Katie et al. [63], using the internet for health purposes was associated with increased depression in the general population. The increase may be due to increased rumination, unnecessary alarm, or over-attention to health problems.

In contrast, use of the internet for communication with friends and family is associated with declines in depression [63] showing that social support is beneficial for well-being and lends support to the idea that the internet is a way to strengthen and maintain social ties. While social media has risks in the form of enabling unhealthy comparisons to others, bullying, or exposure to negative content, it can also have positive influences. Researchers have argued that communication on the internet augments social resources by providing an added avenue for interaction and is associated with better psychological functioning, lower stress and, greater positive effect. Young adults have reported feeling that it helps them to keep in touch with others, strengthen relationships with friends, and allows them to explore new information and perspectives $[13,15]$. Similar to these findings $12 \%$ of the people indicated that screen time has a positive effect on their mental health. Social media platforms offer an opportunity to ameliorate social isolation and thus, digital tech can help overcome challenges during this tumultuous time of social distancing.

P15: "It gives a positive effect on mental health and happiness to forget about ongoing crisis".

On the other end, some students (22\%) reported social isolation and also a strain in interpersonal relations. Technology is now driving our life values and smartphones are actually changing social relationships. Social isolation thus leads to the second issue-whether this new way of being online is going to make people more present in the virtual world at the expense of the real world?

It's worth mentioning that existing literature concerning social media use have shown mixed results. In the context of this variability various factors such as social support garnered through screen-based activities [64], types of social interactions [65], pre-existing levels of social anxiety [66], and fear of missing out [67] might be more influential in predicting well-being outcomes than screen time directly.

However, since we did not control the uses of the internet with regards to the nature of the online resources chosen by the respondents, we cannot further determine the association of media content and its effect on mental health. $59.3 \%$ people reported no change in their mental health because of screen use.

When anxiety is caused by trauma, such as post-traumatic stress disorder, it's not uncommon for the individual to relive that trauma through a vivid, unsettling dream that jerks them out of sleep [68]. For those with panic anxiety disorder, it's not uncommon for them to experience nocturnal panic attacks which take place during the lighter stages of sleep. The individual wakes up feeling extreme panic or fear, perhaps accompanied by sweats, pains in the chest, and increased heart rate. In the present study, 4.7\% students reported such symptoms and thus warrant urgent and effective intervention. It is possible that this pandemic crisis is perceived as a traumatic event by the students.

In addition, to determine the effect of screen use and also response to stress, there are many factors which operate at an individual level such as the psychological make-up of the individual (resilience, coping, and optimism). Thus, identifying predisposing individual traits and characteristics of the technology use may help discern students whose technology habits most likely indicate problematic use and who are emotionally vulnerable and thus susceptible to technology overuse.

\subsection{Physical Health and Well Being}

As well as negatively impacting the adult sleep cycle and mental health, using screens can also affect one's physical health. According to our study, $48 \%$ of the students believe that excessive hours spent in front of the screen 
have affected their physical well being. 87.3\% of participants reported one or more health symptoms as shown in Table 3. A 2012 Journal of Preemptive Medicine study found those who practice a combination of low physical activity and high amounts of screen time were far more likely to rate their health as "poor" [29].

Majority of the student participants reported a change in their sleeping pattern and quality and also encountered various sleeping problems as summarized in Table 4 . Around 52\% people admitted that excessive screen use has affected their sleep quality and linked it to sleeping problems such as reduced sleep durations with mobile phone use-awakenings. When faced with anxiety or stress, $40 \%$ reported decreased sleeping hours, $33.3 \%$ sleep less and $1.4 \%$ of the population experience irregular sleeping patterns.

Table 3. General health problems reported by the participants

\begin{tabular}{cc}
\hline Symptom & Percentage of participants \\
\hline Lack of concentration & $60.7 \%$ \\
Tiredness & $44 \%$ \\
Lack of energy & $42 \%$ \\
Eye strain & $32 \%$ \\
Headache & $31.3 \%$ \\
Weight gain & $29.3 \%$ \\
Exhaustion & $26 \%$ \\
Body pain & $22.7 \%$ \\
None & $12.7 \%$ \\
\hline
\end{tabular}

Table 4. Sleep problems reported by the participants

\begin{tabular}{cc}
\hline Sleep problems & Percentage of participants \\
\hline Sleep more & $53.3 \%$ \\
Sleep less & $4.7 \%$ \\
Altered sleep-wake cycle & $56.7 \%$ \\
Irregular sleep-wake cycle & $30.7 \%$ \\
Problems to fall asleep & $31.3 \%$ \\
Restless sleep & $16.7 \%$ \\
Involuntary awakenings during night & $12 \%$ \\
Too early morning awakenings & $8.7 \%$ \\
Awake from sleep with palpitations in chest feeling heart pound or beat irregularly & $4.7 \%$ \\
Need to take medication for sleeping & $1.3 \%$
\end{tabular}

Moreover, university students who do not get enough sleep tend to engage in social media like Facebook more than their rested peers [69], which further aggravates the problem of technology displacing sleep. Being tired can further increase social media use, which can lead to a vicious circle of reduced sleep and increased social media use.

As previously discussed, sleep and screen time are heavily impacted by the other and can lead to affecting one's behavior as well. If someone does not get an adequate amount of sleep, it can affect their behavior and performance for the day. Students reported feeling of daytime tiredness (44\%), more exhaustibility (26\%), lack of energy (42\%) along with less motivation (65.3\%). These symptoms reported by student participants could be interfering not only with their normal daily activities but with academic performance as well (Effect of screen time use on academic performance of physiotherapy students is conducted along with this study, results of which are presented elsewhere). A Saudi Arabian study found that $44.4 \%$ of the medical student participants attributed their headaches, decreased 
concentration, memory loss, hearing loss, and fatigue to the use of their mobile phones.

Digital eye strain is the most frequent symptom linked to technology addiction and includes dry eyes, headaches, blurred vision, burning, itching, difficulty focusing and pain in the neck and shoulders. In the present survey, 32\% of participants reported eyestrain and headaches. Reading the smaller fonts on a smartphone and tablet devices can cause significantly more eye strain as compared to larger screen based devices such as laptops and computers. A few key considerations around technology use can help to counteract the side effects caused by its overuse. Some of these include taking a "20-20-20" break (taking a 20 second break every 20 minutes to focus on a 20 feet distant object). In this study, only $22.7 \%$ of participants practiced intermittently focusing on distant objects. Also, predominant use of small screen devices, nocturnal use, and inadequate precautionary measures as discussed previously could be the contributing factors. Body pain was reported by (22.7\%) having experienced it newly during the lockdown. 39.3\% practiced stretching in between and $28.7 \%$ maintaining good posture while using digital devices.

It has been postulated that electronic media use may result in less physical activity and higher body mass index [70] [71]. An increased risk of weight gain and obesity is a common result of spending great amounts on screens. This is largely attributed to the sedentary behavior due to the nature of most electronic activities. Sitting to watch television, playing computer games or scrolling on your phone takes time away from physical activities. Also another operating mechanism could be increased eating while using screens, leading to greater calorie intake; a higher night time eating has also been reported. Weight gain during lock down was reported by $29.3 \%$ of the participants. As a good practice, $24.7 \%$ mentioned that they avoid eating in front of the screen.

There is evidence that higher levels of screen time is associated with a variety of health harms for young people, with evidence strongest for adiposity, unhealthy diet, depressive symptoms and quality of life [72].

According to our survey, on an average $97 \%$ of the students have had no exposure to alcohol, smoking/second hand smoking or any kind of medication or drug consumption that affects one's physical well-being or sleep quality. However, 39.3\% of the students have had regular exposure to caffeine and also this could have acted as a confounding factor for this part of the sample. Low intake of caffeine is known to have minimal effect on physical well-being. To measure the quantity of exposure to these factors is beyond the scope of this study. Future studies should consider controlling these variables.

On its other aspect, during the COVID-19 lockdown, exposure to alcohol, smoking/second hand smoking, caffeine has significantly increased. The links between traumatic stress and drug use are well-established, "People respond in the usual ways, such as painkillers, alcohol and recreational drugs". Worrying figures from global analytics company Nielsen revealed that sales of alcohol have increased 291\% during the lockdown [73]. Researchers at the University of Portsmouth fear a spike in alcohol misuse during lockdown that may result in longer-term health issues for at-risk individuals and have launched a study to investigate this. "This period of isolation might lead to a spike in alcohol misuse and, potentially, development of addiction in at-risk individuals or relapse in recovered addicted patients, therefore placing further strain on the health service in general, during and after the pandemic”.

Previous studies on university students have revealed that students indulge in alcohol or substance use as a stress coping strategy. A recently published study has revealed that the adverse consequences such as increased stress and social isolation following COVID-19 related University closings resulted in increased alcohol consumption in students [74]. In the current study, though psychological distress was experienced by the students, such negative behavior was not observed. However, we could not rule out under reporting of such behavior by students in spite of assurance of anonymity and confidentiality of their responses by the investigators involved in the present study.

We exert caution in interpreting this relationship as we cannot separate the smartphone usage time from the influence of disturbed sleep patterns on mental well-being and general health of the participants. There's no denying sleep and mental health have a reciprocal relationship. Lack of sleep impacts mental health, and vice versa. The worst part is, sleep deprivation and stress contribute to a negative feedback loop that can be difficult to break and often results in mood disorders, anxiety disorders, and depressive disorders.

\subsection{Physical Exercises}

Many of the concerns around screen use relate to sedentary (or inactive) behavior. The idea being that time spent in front a screen is time that is not spent exercising or doing other forms of physical activity. Sedentary behavior may be associated with poorer physical health, wellbeing, and mental health.

During the lockdown, 65.3\% of the students have been taking part in physical exercises regularly (minimum 3-5 days a week) at home. The type of physical exercises performed by the students included aerobic exercises (44\%), yogasana (27.3\%), pranayama (18.7\%), resistance training (23.4\%), indoor walking (2.6\%).

The study also throws light on the fact that physical exercises could be a mediating factor for effect on sleep pat- 
tern and overall perception of physical well-being. $65.27 \%$ of the exercising participants experienced beneficial effects due to performance of physical exercises and reported decreased sleeping complaints or mental health issues.

A recent meta-analysis of six studies found that exercise training resulted in modest improvements in subjective sleep quality in middle- to older-aged adults with sleep problems.

As a prominent example, Reid and colleagues [75] found that 4 months of aerobic exercise training in a sample of older adults with insomnia significantly improved sleep quality while also reducing daytime sleepiness and depressive symptoms. Thus, exercise may hold the most promise for those with more severe or more chronic sleep disturbance and mental health issues.

The National Sleep Foundation [76] states that regular exercise, particularly in the morning or afternoon, can impact our sleep quality by raising our body temperature a few degrees. Later in the day, when our internal thermostat drops back to its normal range, this can trigger feelings of drowsiness and help us drop off to sleep. Also, if we exercise outdoors, we will be exposed to natural light, an important element in helping our body establish a good sleep-wake cycle.

Physical exercises are also said to reduce or prevent mental health issues like depression, anxiety, stress, etc., aerobic exercises, including jogging, swimming, cycling, walking, gardening, and dancing, have been proved to reduce anxiety and depression [77].

These improvements in mood are proposed to be caused by exercise-induced increase in blood circulation to the brain and by an influence on the hypothalamic-pituitary-adrenal (HPA) axis and, thus, on the physiologic reactivity to stress [77]. This physiologic influence is probably mediated by the communication of the HPA axis with several regions of the brain, including the limbic system, which controls motivation and mood; the amygdala, which generates fear in response to stress; and the hippocampus, which plays an important part in memory formation as well as in mood and motivation. Other hypotheses that have been proposed to explain the beneficial effects of physical activity on mental health include distraction, self-efficacy, and social interaction.

Exercise has also been found to alleviate symptoms such as low self-esteem and social withdrawal. Exercise releases chemicals like endorphins and serotonin that improve your mood. Blumenthal has explored the mood-exercise connection through a series of randomized controlled trials [78]. In one such study, he and his colleagues assigned sedentary adults with major depressive disorder to one of four groups: supervised exercise, home-based exercise, antidepressant therapy or a placebo pill. After four months of treatment, Blumenthal found, patients in the exercise and antidepressant groups had higher rates of remission than did the patients on the placebo. Exercise is especially important in patients with schizophrenia since these patients are already vulnerable to obesity and also because of the additional risk of weight gain associated with antipsychotic treatment, especially with the atypical antipsychotics [77]. Patients suffering from schizophrenia who participated in a 3-month physical conditioning program showed improvements [77].

In agreement with previous studies, the relationship between screen-time behaviors and physical activity is complex and not always consistent [79], which could be attributed to the implication of other uses of the smartphone and other related outcomes (e.g., sleep patterns or other sedentary behaviors) [80]. The identified research indicates screen time has affected sleep, mental and physical health in a particular population and exercise is beneficial for these variables. It is clear that additional research is necessary particularly because of the variance of these findings between study populations and to further explore the benefits pertaining to different types of exercise interventions.

\section{Discussion}

Through this qualitative survey we aimed to analyze the pattern of electronic media use and its impact in Physiotherapy students during COVID-19 pandemic lock down. To the best of our knowledge, this is the first study which has explored the psychological experience of university students in the context of this pandemic lock down. This is one of the few cross sectional studies on electronic media use and mental health and physical well-being which included smartphone use. As an asset of this design, we were able to explore different exposure-response relationships to draw inferences from electronic media use to mental and general health.

The present study contributes to the growing literature highlighting the associations between screen use, in particular sleep outcomes and a wide range of health outcomes in general. The pervasive phenomenon of excessive screen time use has widespread implications due to the associations between problematic screen use and increased risk of physical morbidities, disrupted psychological well-being and impaired cognitive/academic functioning.

Although the smartphone has become one of the most popular and important communication tools, its excessive use has emerged as a social issue worldwide and created a new mental health concern, wherein the user tends to 
develop dependency on it. While researchers continue to study the effects and extent of phone use, the scientific consensus is that phone addiction is real [81].

It has been publicized extensively as an emerging social problem. Even though behavioral addiction does not involve the use of a chemical substance, the similarities between the cardinal features of substance use disorders and behavioral addiction disorders such as smartphone addiction are striking. In general terms, both may be described as disorders involving a loss of control over a compulsive, time- and resource-consuming behavior, which persists despite adverse consequences, characterized by a continued escalation of the behavior, or withdrawal symptoms when engagement in the behavior is reduced.

This addiction is mainly characterized by excessive or poorly controlled preoccupations, urges, or behaviors regarding smartphone use, to the extent that individuals neglect other areas of life [82]. Considering the high rate of smartphone use among university students, this area needs to be further explored, with a focus on what roles technology plays in fostering fantasies, acting out behaviors.

Mental health is a crucial aspect that needs to be addressed during this lock-down as all modes of communication revolve around the virus. Capsized travel plans, indefinite isolation, panic over scarce resources and information overload could be a recipe for unchecked anxiety and feelings of isolation. In addition, people lose face-to-face connections and traditional social interventions, and this is a stressful phenomenon with reduced access to family, friends, and other social support systems causing loneliness and triggering or increasing mental issues like anxiety and depression. It can be inferred from these points that psychological interventions in the face of such crises are part of the health care system in the context of public health emergencies. It is vital to educate and prepare them to face the mental health issues they may endure during the period.

We acknowledge several limitations of the study. Students self-reported information on media use and health variables might generally be biased by recall and subjectivity. In addition, the lack of objective screen time data is of concern. Also, uncertainty due to various psychosocial factors acting dynamically during the pandemic situation has resulted in various mental health issues in the general population. Owing to the complex interaction of the multiple factors viz. screen time, sleep, mental health and physical health we cannot separate the individual contribution of each factor. Excessive screen time as a contributor to mental and physical health thus needs to be interpreted in a broader context. Also the cross sectional nature of the study precludes causal inferences on these associations and limits the possibility to determine changes over time in all these variables. We recommend future longitudinal studies to further explore this dynamic relationship. The survey sample size is not sufficiently large to allow for true population representation. However, this set of findings does accurately represent the views of those contained within the sample of valid survey responses.

\section{Conclusion}

Use of screen based digital devices and media has significantly increased during COVID-19 pandemic lock down in Physiotherapy students. High proportions of university students engage in heavy smartphone use and media multitasking, with resultant chronic sleep deprivation, and negative effects on cognitive control, academic performance and socio-emotional functioning. The present study strengthens the existing scientific evidence for the negative impact of excessive screen time on mental and physical health while providing new insights on the digital media use during pandemic situation. In addition, this study also attempts to determine the mediating effect of physical exercise on ill effects of screen use. This study can be considered as a way to bring awareness about the adverse repercussions of increased screen usage and a preliminary step to identify its effects on the psychological outcomes of physiotherapy students.

\section{Implications}

Students obligated to view screens for a means of studies may not be able to use screen time less than recommended, but there are other recommendations that help mitigate negative health effects. Instead, we encourage smart use of technology that takes advantage of its conveniences and counteracts the side effects caused by overuse. Through these study findings, we aim to implement better and healthier practices by sensitizing students about the negative effects, encouraging better bedtime routines, and limiting the device use for academic purposes with alternative sources and strategies. The impact of mobile use on psychology and health should be discussed among the students. The prevalence of mental health issues identified in this study supports the need to expand mental health services to meet the special needs of vulnerable population.

We would like to conclude with a statement by a participant

P35: "Some entertainment with learning should be promoted for being mentally healthy and studying at the same 
time, especially during this crisis".

We recommend students to utilize the screen time productively.

\section{Acknowledgement}

The authors would like to acknowledge the valuable contribution of student participants across Maharashtra and support of the principal and faculty of the institute in the conduct of the study.

\section{Conflict of interest}

The authors declare that they have no conflict of interests.

\section{References}

[1] Telecom Regulatory Authority of India. (2016). Telecom Subscription Data as on 30th April, 2016. Ministry of Telecommunication. Government of India.

[2] Bagozzi, R. P. (2007). The legacy of the technology acceptance model and a proposal for a paradigm shift. J Assoc Inf Syst. 8: 244-54.

[3] Elogie, A. A. (2015). Factors influencing the adoption of smartphones among undergraduate students in Ambrose Alli University, Ekpoma, Nigeria. Libr Philos e J 2015. Paper 1257. [Last accessed on May 18, 2017]. Available from: http://www.digitalcommons.unl.edu/libphilprac/1257.

[4] Boruff, J. T., Boruff, D. (2014). Mobile devices in medicine: a survey of how medical students, residents, and faculty use smartphones and other mobile devices to find information. J Med Libr Assoc. 2014 Jan; 102(1): 22-30.

[5] Greenhalgh, T. (2001). Computer assisted learning in undergraduate medical education. BMJ. 2001; 322(7277): 40-44. [PMC free article] [PubMed] [Google Scholar]

[6] Bridge, P. D., Jackson, M., Robinson L. (2009). The effectiveness of streaming video on medical student learning: a case study. Med Educ Online. 2009; 14: 11. Available at: http://www.ncbi.nlm.nih.gov/pmc/articles/PMC2779626/.

[7] Wong, G., Greenhalgh T., Pawson R. (2010). Internet-based medical education: a realist review of what works, for whom and in what circumstances. BMC Med Educ. 2010; 12 https://doi.org/10.1186/1472-6920-10-12.

[8] Twenge, J. M. (2019). More time on technology, less happiness? Associations between digital-media use and psychological well-being. Curr. Dir. Psychol. Sci. 2019, 28, 372-379. [CrossRef]

[9] Twenge, J. M., Campbell, W. K. (2018). Associations between screen time and lower psychological well-being among children and adolescents: Evidence from a population-based study. Prev. Med. Rep. 2018, 12, 271-283.

[10] Odgers, C. L., Jensen, M. R. (2020). Annual Research Review: Adolescent Mental Health in the Digital Age: Facts, Fears and Future Directions. J. Child. Psychol. Psychiatry 2020, 61, 336-348.

[11] Orben, A. (2020). Teenagers, Screens and Social Media: A Narrative Review of Reviews and Key Studies. Soc. Psychiatry Psychiatr. Epidemiol. 2020, 55, 407-414.

[12] Jensen, M., George, M. J., Russell, M. R., Odgers, C. L. (2019). Young adolescents' digital technology use and mental health symptoms: Little evidence of longitudinal or daily linkages. Clin. Psychol. Sci. 2019, 7, 1416-1433. [CrossRef]

[13] Orben, A., Dienlin, T., Przybylski, A. K. (2019). Social Media’s Enduring Effect on Adolescent Life Satisfaction. Proc. Natl. Acad. Sci. USA 2019, 116, 21. [CrossRef]

[14] Orben, A., Przybylski, A. K. (2019). The association between adolescent well-being and digital technology use. Nat. Hum. Behav. 2019, 3, 173-182.

[15] American Psychiatric Association. Diagnostic and statistical manual of mental disorders.

[16] Young, K. S. (1998). Internet addiction: The emergence of a new clinical disorder. Cyberpsychol Behav. 1998; 1: $237-44$.

[17] Griffiths, M. (2005). A ‘components’ model of addiction within a biopsychosocial framework. J Subst Use. 2005 ; 10 : $191-7$.

[18] Park N, Lee H. (2014). Nature of youth smartphone addiction in North Korea: Diverse dimensions of smartphone use and individual traits. J Commun Res 2014; 51: 100-132.

[19] Nikhita C. S., Jadhav P. R., Ajinkya S. (2015). A. Prevalence of mobile phone dependence in secondary school adolescents. J Clin Diagn Res 2015; 9: VC06-9.

[20] 13th edition of Digital media trends survey, conducted by Deloitte's Technology, Media \& Telecommunications practice.

[21] https://www.nielsen.com/us/en/insights/report/2018/q1-2018-total-audience-report/.

[22] Mangot, A. G., Murthy, V. S., Kshirsagar, S. V., Deshmukh, A. H., Tembe, D. V. (2018). Prevalence and Pattern of Phantom Ringing and Phantom Vibration among Medical Interns and their Relationship with Smartphone Use and Perceived Stress. Indian J Psychol Med. 2018 Sep-Oct; 40(5): 440-445. 
[23] Nowreen, N., Ahad F. (2018). Effect of smartphone usage on quality of sleep in medical students. Natl J Physiol Pharm Pharmacol. 2018; 8: 1366-70.

[24] Prasad S., Harshe D., Kaur N., Jangannavar S., Srivastava A., Achanta U., Khan S., Harshe G. (2018). A Study of Magnitude and Psychological Correlates of Smartphone Use in Medical Students: A Pilot Study with A Novel Telemetric Approach. Indian J Psychol Med. 2018 Sep-Oct; 40(5): 468-475.

[25] Aggarwal M, Grover S, Basu D. (2012). Mobile phone use by resident doctors: Tendency to addiction-like behavior. German J Psychiatry 2012; 15: 50-5.

[26] Munezawa T., Kaneita Y., Osaki Y., Kanda H., Minowa M., Suzuki S., Higuchi S., Mori J., Yamamoto R., Ohida T. (2011). The association between use of mobile phones after lights out and sleep disturbances among Japanese adolescents: a nationwide cross-sectional survey. Sleep 2011; 34: 1013-1020.

[27] Brauser D. (2013). Smartphone ‘Addiction’ May Affect Adolescent Development. [Last updated on 2013 May 23; Last cited on 2014 Jan 21]. Available from: http://www.medscape.com/viewarticle/804666.

[28] Flanagan J. (2013). “Smart phone ‘addicts' do not need rehab” The Kernel. [Last updated on 2013 Oct 22; Last cited on 2013 Nov 1]. Available from: http://www.kernelmag.com/features/report/6240/smart-phone-addicts-do-not-need-rehab.

[29] K. C. Madhava, Shardulendra Prasad Sherchand, Samendra Sherchan. (2017). Association between screen time and depression among US adults. Preventive Medicine Reports.Volume 8, December 2017, Pages 67-71.

[30] Ohayon M. M., Roth T. Place of chronic insomnia in the course of depressive and anxiety disorders. J Psychiatric Res. 2003;37: 9-15

[31] https://thoracickey.com/sleep-eeg/.

[32] National Centre for Health Statistics. (1970). Selected Symptoms of Psychological Distress. US Public Health Service Publication 1000, Series 11, Number 37. Washington, DC: US Department of Health, Education and Welfare; 1970.

[33] Colten H. R., Altevogt B. M. (2006). Sleep Disorders and Sleep Deprivation: An Unmet Public Health Problem. Institute of Medicine (US) Committee on Sleep Medicine and Research; 2006.

[34] Bixler E. O., Kales A., Soldatos C. R., Kales J. D., Healy S. (1976). Prevalence of sleep disorders in the Los Angeles metropolitan area. Am J Psychiatry. 1979; 136: 1257-1262. [PubMed] [Google Scholar]

[35] Mellinger G. D., Baiter M. B., Uhlenhut E. H. (1985). Insomnia and its treatment. Prevalence and correlates. Arch Gen Psychiatry. 1985; 42: 225-232. [PubMed] [Google Scholar]

[36] Ohayon M. M. (1996). Epidemiological study on insomnia in a general population. Sleep. 1996; 57. [Google Scholar]

[37] C. A. Brick, D. L. Seely, T. M. Palermo. (2010). Association between sleep hygiene and sleep quality in medical students. Behav Sleep Med, 8 (2010), pp. 113-121, 10.1080/15402001003622925.

[38] E. Preišegolavičiūtè, D. Leskauskas, V. Adomaitienè. (n.d.). Associations of quality of sleep with lifestyle factors and profile of studies among Lithuanian students.

[39] H. M. Abdulghani, N. A. Alrowais, N. S. Bin-Saad, N. M. Al-Subaie, A. M. Haji, A. I. (2012). Alhaqwi. Sleep disorder among medical students: relationship to their academic performance. Med Teach, 34 (Suppl 1) (2012), pp. S37-S41, 10.3109/0142159X.2012.656749.

[40] A. S. Bahammam, A. M. Alaseem, A. A. Alzakri, A. S. Almeneessier, M. M. Sharif. (2012). The relationship between sleep and wake habits and academic performance in medical students: a cross-sectional study. BMC Med Educ, 12 (2012), p. 61, 10.1186/1472-6920-12-61.

[41] Foerster M., Henneke A., Chetty-Mhlanga S., Röösli M. (2019). Impact of Adolescents’ Screen Time and Nocturnal Mobile Phone-Related Awakenings on Sleep and General Health Symptoms: A Prospective Cohort Study. Int. J. Environ. Res. Public Health 2019, 16, 518; doi:10.3390/ijerph16030518.

[42] Gradisar M., Wolfson A. R., Harvey A. G., Hale L., Rosenberg R., Czeisler C. A. (2013). The sleep and technology use of Americans: fndings from the National Sleep Foundation's 2011 Sleep in America Poll. J Clin Sleep Med 2013; 9(12): 1291-1299.

[43] Michael M, Colin B, Mika K. (2000). The health hazards of mobile phones. Br Med J 2000; 20: 1288-9.

[44] Bortkiewicz A. (2001). A study on the biological effects of exposure to mobile phone frequency EMF. Med Pr 2001; 52(2): 101-6.

[45] Jamal, A., Sedie, R., Haleem, K., and Hafiz, Najla. (2012). Patterns of use of 'smart phones' among female medical students and self-reported effects. Journal of Taibah University Medical Sciences. 7. 45-49. 10.1016/j.jtumed.2012.07.001.

[46] Lockdown has affected mental health of many—what needs to be done to avoid panic. (2020). Published: May 23, 2020. Financial Express: Read to Learn.

[47] SADAG's Online Survey Findings on COVID-19 and Mental Health. (2020). Published: April 21, 2020. Available on: https://www.polity.org.za/article/sadags-online-survey-findings-on-covid-19-and-mental-health-2020-04-21.

[48] Schmitt, B., Gugger, M., Augustiny, K., Bassetti, C., Radanov, Bogdan. (2000). Prevalence of sleep disorders in an employed 
Swiss population: results of a questionnaire survey. Schweizerische medizinische Wochenschrift. 130. 772-8.

[49] Definition of SCREEN TIME. (n.d.). www.merriam-webster.com. Retrieved 9 November 2019.

[50] Winther D., Byrne J. (2020). Rethinking screen-time in the time of COVID-19, UNICEF 07 April 2020 https://www.unicef.org/globalinsight/stories/rethinking-screen-time-time-covid-19.

[51] Chanchani M., Mishra D. (2020). Mobile apps' usage spikes in lockdown, The times of India Business, April 15, 2020. https://timesofindia.indiatimes.com/business/india-business/mobile-apps-usage-spikes-in-lockdown/articleshow/75148768.cms.

[52] Brand Wagon Online Amazon and Netflix witnessed more than 60\% growth in subscriber base during lockdown: Velocity MR Study, Financial Express, 20 May 2020, https://www.financialexpress.com/brandwagon/amazon-and-netflix -witnessed-more-than-60-growth-in-subscriber-base-during-lockdown-velocity-mr-study/1965362/

[53] Goodman A. (1990). Addiction: Definition and implications. Br J Addict. 1990; 85: 1403-8.

[54] Domoff, S. E., Borgen, A. L., Foley, R. P., \& Maffett, A. (2019). Excessive use of mobile devices and children’s physical health. Human Behavior and Emerging Technologies, 2, 169-175.

[55] J. S. Lemmens, P. M. Valkenburg, D. A. Gentile. (2015). The internet gaming disorder scale Psychological Assessment, 72(2015), pp. 567-582. http://dx.doi.org/10.1037/pas0000062.

[56] R. J. J. M. Van den Eijnden, G. Meerkerk, A. A. Vermulst, R. Spijkerman, R. C. M. E. Engels. (2007). Online communication, compulsive Internet use, and psychosocial well-being among adolescents: a longitudinal study. Developmental Psychology, 44 (2007), pp. 655-665.

[57] Wilcockson, T. D. W., Osbourne, A. M., Ellis, D. A. (2019). Digital detox: The effect of smartphone abstinence on mood, anxiety, and craving. Addict. Behav. 2019, 99, 106013.

[58] Elhai, J. D., Yang, H., Montag, C. (2019). Cognitive- and Emotion-Related Dysfunctional Coping Processes: Transdiagnostic Mechanisms Explaining Depression and Anxiety’s Relations with Problematic Smartphone Use. Curr Addict Rep 6, 410-417 (2019). https://doi.org/10.1007/s40429-019-00260-4.

[59] L. de Wit, A. van Straten, F. Lamers, P. Cuijpers, B. Penninx. (2011). Are sedentary television watching and computer use behaviors associated with anxiety and depressive disorders? Psychiatry Res., 186 (2-3) (2011), pp. 239-243, 10.1016/j.psychres.2010.07.003.

[60] Xiaoyan Wu, Shuman Tao, Shichen Zhang, Yukun Zhang, Kaihua Chen, Yajuan Yang, Jiahu Hao, Fangbiao Tao. (2016). Impact of screen time on mental health problems progression in youth: a 1-year follow-up study. BMJ Open. 2016; 6(11): e011533. Published online 2016 Nov 9. doi: 10.1136/bmjopen-2016-011533.

[61] https://www.premierhealth.com/your-health/articles/health-topics/screen-addiction-affects-physical-and-mental-health.

[62] Panger GT. Emotion in Social Media. (2017). http://people.ischool.berkeley.edu/ gpanger/dissertation/emotion_in_social_media.pdf.

[63] Katie Bessière, Sarah Pressman, Sara Kiesler, Robert Kraut. (2010). Effects of Internet Use on Health and Depression: A Longitudinal Study. J Med Internet Res. 2010 Jan-Mar; 12(1): e6. Published online 2010 Mar 12. doi: 10.2196/jmir.1149.

[64] Wang, P., Lei, L., Wang, X., Nie, J., Chu, X., Jin, S. (2018). The exacerbating role of perceived social support and the "buffering” role of depression in the relation between sensation seeking and adolescent smartphone addiction. Pers. Individ. Dif. 2018, $130,12$.

[65] Twenge, J. M., Spitzberg, B. H., Campbell, W. K. (2019). Less in-person social interaction with peers among US adolescents in the 21st century and links to loneliness. J. Soc. Pers. Relat. 2019, 36, 1892-1913.

[66] Hong, W., Liu, R., Oei, T., Zhen, R., Jiang, S., Sheng, X. (2019). The mediating and moderating roles of social anxiety and relatedness need satisfaction on the relationship between shyness and problematic mobile phone use among adolescents. Comput. Hum. Behav. 2019, 93, 301-308.

[67] Elhai, J. D., Levine, J. C., Dvorak, R. D., Hall, B. J. (2016). Fear of missing out, need for touch, anxiety and depression are related to problematic smartphone use. Comput. Hum. Behav. 2016, 63, 509-516.

[68] Nielsen T, Levin R. (2007). Nightmares: a new neurocognitive model. Sleep Med Rev. 2007; 11(4): 295.

[69] Mark, G., Wang, Y., Niiya, M., Reich, S. (2016). Sleep Debt in Student Life: Online Attention Focus, Facebook, and Mood. 5517-5528. 10.1145/2858036.2858437.

[70] David S. Bickham, Emily A. Blood, Courtney E. Walls, Lydia A. Shrier, Michael Rich. (2013). Characteristics of Screen Media Use Associated With Higher BMI in Young Adolescents Pediatrics. 2013 May; 131(5): 935-941. doi: 10.1542/peds.2012-1197.

[71] Cha E. M., Hoelscher D. M., Ranjit N., Chen B., Gabriel K. P., Kelder S., et al. (2018). Effect of Media Use on Adolescent Body Weight. Prev Chronic Dis 2018; 15: 180206. DOI: http://dx.doi.org/10.5888/pcd15.180206external.

[72] Stiglic N., Viner R. M. (2019). Effects of screentime on the health and well-being of children and adolescents: a systematic review of reviews. BMJ Open. 2019; 9(1): e023191. Published online 2019 Jan 3. doi: 10.1136/bmjopen-2018-023191.

[73] https://www.nielsen.com/us/en/insights/article/2020/rebalancing-the-covid-19-effect-on-alcohol-sales/. 
[74] Lechner W. V., Laurene K. R., Patel S., Megan Andersonb M., Gregab C., Kenneb D. R. (2020). Changes in alcohol use as a function of psychological distress and social support following COVID-19 related University closings. Addict Behav. 2020 11; 110: 106527.

[75] Reid, K. J., Baron, K. G., Lu, B., Naylor, E., Wolfe, L. and Zee, P. C. (2010). Aerobic exercise improves self-reported sleep and quality of life in older adults with insomnia. Sleep Med., 2010, 11: 934-940.

[76] National Sleep Foundation. How Exercise Impacts Sleep Quality. Available at: https://www.sleepfoundation.org/articles/how-exercise-impacts-sleep-quality.

[77] Sharma A., Madaan V., Petty F. D. (2006). Exercise for Mental Health. Prim Care Companion J Clin Psychiatry. 2006; 8(2): 106. doi: 10.4088/pcc.v08n0208a.

[78] Blumenthal J. A., Babyak M. A., Doraiswamy, M., Watkins L., Hoffman B. M., Barbour A. K., et al. (2007). Exercise and Pharmacotherapy in the Treatment of Major Depressive Disorder. Psychosom Med. 2007; 69(7): 587-596.

[79] Fobian A. D., Avis K., Schwebel D. C. (2016). Impact of media use on adolescent sleep efficiency. J. Dev. Behav. Pediatr. 2016; 37: 9-14. doi: 10.1097/DBP.0000000000000239.

[80] Perrault A. A., Bayer L., Peuvrier M., Afyouni A., Ghisletta P., Brockmann C., Spiridon M., Hulo Vesely S., Haller D. M., Pichon S., et al. (2019). Reducing the use of screen electronic devices in the evening is associated with improved sleep and daytime vigilance in adolescents. Sleep. 2019; 42: 125. doi: 10.1093/sleep/zsz125.

[81] De-Sola Gutiérrez, J de Fonseca, F. R. Rubio G. (2016). Cell-Phone Addiction: A Review. Front Psychiatry. 2016; 7: 175. doi: 10.3389/fpsyt.2016.00175.

[82] W. Jun. (2015). “An Analysis Study on Correlation of Internet Addiction and School Age”. 2015 2nd International Conference on Information Science and Security (ICISS), Seoul, 2015, 1-3. doi: 10.1109/ICISSEC.2015.7370977. 